\title{
SEMIGROUP-GRADED RINGS WITH FINITE SUPPORT
}

\author{
by M. V. CLASE, E. JESPERS and A. DEL RÍO
}

(Received 29 April, 1994; revised 15 August, 1994)

1. Introduction. Let $S$ be a semigroup and let $R=\bigoplus_{S \in S} R_{s}$ be an $S$-graded ring. If $R_{s}=0$ for all but finitely many elements $s \in S$, then $R$ is said to have finite support. In this paper we concern ourselves with the question of whether a graded ring $R$ with finite support inherits a given ring theoretic property from the homogeneous subrings $R_{e}$ corresponding to idempotent semigroup elements $e$.

When $S$ is finite, an approach based on the structure theory of semigroups has led to an affirmative answer to this question for perfect, semilocal and semiprimary rings [3], for Jacobson rings [4], and for PI-rings [11].

If $S$ is an arbitrary semigroup and $R$ has finite support, then structure theoretic methods at the semigroup level require dealing with principal factors which are 0 -simple but not completely 0 -simple. This approach leads to difficulties because the structure of such factors is not well understood. In the absence of such factors, the proofs developed for finite semigroups can be easily modified to handle the finite support case [2].

In this paper, we present a new method to deal with graded rings with finite support. For such rings, we obtain results of the form: if each subring $R_{e}, e$ an idempotent in the support, is a perfect ring, then $R$ is a perfect ring. The method relies on the class of rings under consideration (for example, perfect rings) being closed under various constructions (cf. Lemma 2); then induction on the size of the support is used to reduce to the case of a ring graded by a finite group.

This method avoids use of the structure theory of semigroups almost entirely, only requiring a simple result that a semigroup satisfying a certain property is in fact a finite group. Note also that the results obtained include the cases of rings graded by finite semigroups and group-graded rings with finite support.

For group-graded rings, there are many examples in the literature of results which hold for finite group-graded rings remaining true for group-graded rings with finite support (see, for example [1], [7], [12], [13], [14], [15]). However, each such result requires a separate method of proof; there seems to be no obvious structural reason why results can be extended in this way. The current paper, using ideas from semigroupgraded rings, provides a more general approach which perhaps clarifies why results extend from finite group-graded rings to graded rings with finite support.

We will give the proofs in detail for perfect rings, but our method works for semiprimary, semilocal, Jacobson, nilpotent, and PI-rings.

The results we obtain for semilocal and perfect rings complement nicely some recent results of Jespers and Okniński [10] which show that in many cases, a perfect (or semilocal), group- or semigroup-graded ring is, modulo its graded Jacobson radical, a graded ring with finite support.

This research was completed while the first author visited Universidad de Murcia whom he would like to thank for their support and hospitality. The second author is supported in part by NSERC-grant OGP0036631 and DGICYT, Spain. The third author is supported in part by DGICYT, PB90-0300-02-02.

Glasgow Math. J. 38 (1996) 11-18. 
Of course, this work raises the question of whether an $S$-graded ring with finite support could not somehow be regraded by a finite semigroup $S^{\prime}$, or at least a semigroup in which all 0-simple principal factors were completely 0-simple. If this were always possible, then the methods of [2], [3], [4], [11] suffice. We provide examples to show that this is not so.

2. Preliminaries. All rings are associative and do not necessarily have an identity element.

Write $J(R)$ for the Jacobson radical of a ring $R$. A ring $R$ is right $T$-nilpotent if for every sequence $x_{1}, x_{2}, x_{3}, \ldots$ of elements of $R$, there is an $n$ such that $x_{n} x_{n-1} \ldots x_{1}=0$. We define left $T$-nilpotent similarly.

A ring $R$ is semilocal if $R / J(R)$ is Artinian. $R$ is semiprimary if it is semilocal and $J(R)$ is nilpotent. $R$ is right perfect if it is semilocal and $J(R)$ is right T-nilpotent; left perfect is similarly defined. We shall use perfect to mean right perfect throughout, but all results obtained hold equally for left perfect rings.

A ring $R$ is a Jacobson ring if $J(R / P)=0$ for every prime ideal $P$ of $R$.

Let $S$ be a semigroup. An $S$-graded ring is a ring $R$ together with a direct sum decomposition (as an additive group) $R=\bigoplus_{s \in S} R_{s}$ with the property that $R_{s} R_{t} \subseteq R_{s t}$ for all $s, t \in S$. The summands $R_{s}$ are called homogeneous components and elements of these summands are called homogeneous elements.

The support of $R$ is the set $\operatorname{supp}(R)=\left\{s \in S \mid R_{s} \neq 0\right\}$. We say that $R$ has finite support if $\operatorname{supp}(R)$ is a finite set.

Let $I$ be an ideal of $R$. For $s \in S$, let $I_{s}=I \cap R_{s}$. If $I=\sum_{s \in S} I_{s}$, then $I$ is a homogeneous ideal. In this case, $I=\bigoplus_{s \in S} I_{s}$ and $R / I=\bigoplus_{s \in S} R_{s} / I_{s}$ are $S$-graded rings. We may similarly define homogeneous left and right ideals.

If $A$ is a subset of $R$, then $R^{1} A$ denotes the left ideal generated by $A$, and similarly, $A R^{1}$ and $R^{1} A R^{1}$ denote the right and two-sided ideals generated by $A$. Note that a oneor two-sided ideal generated by homogeneous elements is necessarily homogeneous.

3. Perfect rings. We will prove the following theorem to illustrate our method.

THEOREM 1. Let $S$ be a semigroup and let $R$ be an $S$-graded ring with finite support. If $R_{e}$ is a (right) perfect ring for each idempotent $e$ in $\operatorname{supp}(R)$, then $R$ is a (right) perfect ring.

The properties that we require of the class of perfect rings are summarised in the following lemma. Proofs of these facts can be found in [1], [2], [3].

LEMMA 2.

(i) A nilpotent ring is perfect.

(ii) A left or right ideal of a perfect ring is perfect.

(iii) Let $R=R_{1}+R_{2}+\ldots+R_{n}$ where each $R_{i}$ is a left (resp. right) ideal of $R$. If each $R_{i}$ is a perfect ring, then $R$ is perfect.

(iv) $A$ homomorphic image of a perfect ring is perfect.

(v) Let $I$ be an ideal of a ring $R$. If I and $R / I$ are perfect, then $R$ is perfect.

(vi) Let $R$ be a ring graded by a finite group $G$. If $R_{1}$ is perfect then $R$ is perfect. 
As will be apparent from the proof of Theorem 1, these are the only properties required of the class of perfect rings. So for any class of rings with a similar set of closure properties, we can obtain similar results. In particular, we have the following result.

Proposition 3. Results analogous to Theorem 1 hold for the classes of semilocal, semiprimary, Jacobson, nilpotent, and PI-rings.

Verifications of the appropriate closure properties which are not straight-forward can be found in [1], [2], [3] for semilocal and semiprimary rings, in [6] for nilpotent rings, and in [11], [17] for PI-rings. The class of Jacobson rings is a left and right hereditary radical class [9], [18] from which fact analogues of properties (ii), (iv), and (v) are immediate. The other properties of Jacobson rings are verified in [2], [4], [16]. (Note that [4] proves (iii) for Jacobson rings in the case that $R$ is a direct sum of a finite set of its right ideals: such a ring is graded by a left zero .semigroup with the ideals as homogeneous components. When the sum in (iii) is not direct, the additive direct sum $\bar{R}=R_{1} \oplus R_{2} \oplus$ $\ldots \oplus R_{n}$ can be made into a ring by defining multiplication on homogeneous components $R_{i}$ and $R_{j}$ to be the multiplication in $R$, and extending this distributively to all of $\bar{R}$. The original ring $R$ is then a homomorphic image of $\bar{R}$.)

Proof of Theorem 1. Let $S$ be a semigroup and let $R$ be an $S$-graded ring with finite support such that $R_{e}$ is perfect for every idempotent $e \in S$. Let $X=\operatorname{supp}(R)$. We will proceed by induction on $n=|X|$.

If $n=1$, then $X=\{x\}$ for some $x$, and $R=R_{x}$. If $x$ is idempotent, then $R$ is perfect by hypothesis. Otherwise, $R^{2}=0$ and $R$ is perfect by (i).

Assume that the result is true for rings with fewer than $n$ elements in the support.

Suppose that there is an $x \in X$ such that $\left|\operatorname{supp}\left(R^{1} R_{x}\right)\right|<n$. Then for all $y \in X$, we have $\left|\operatorname{supp}\left(R^{1} R_{x} R_{y}\right)\right|<n$ as well. Consider the $\operatorname{ring} A=R^{1} R_{x}$. Let $e$ be an idempotent of $S$. If $x=e$, then $A_{e}=R_{e}$ is perfect. Otherwise, $A_{e}=\sum_{z x=e} R_{z} R_{x}$, which is a left ideal of $R_{e}$ and is therefore perfect by (ii). By induction, the ring $A=R^{1} R_{x}$ is perfect. A similar argument shows that each ring $R^{1} R_{x} R_{y}$ is perfect.

Let $I=R^{1} R_{x} R^{1}$. Since $I=R^{1} R_{x}+\sum_{y \in X} R^{1} R_{x} R_{y}, I$ is perfect by (iii). Since $I$ is a homogeneous ideal of $R$, the quotient $\bar{R}=R / I$ is also an $S$-graded ring. For each idempotent $e \in S, \bar{R}_{e}=R_{e} / I_{e}$ is perfect by (iv). Since $R_{x} \subseteq I, \operatorname{supp}(\bar{R})$ is strictly smaller than $X$. So by induction, $\bar{R}$ is perfect. We conclude that $R$ is perfect by (v).

A similar argument holds if $\left|\operatorname{supp}\left(R_{x} R^{1}\right)\right|<n$ for some $x \in X$.

Otherwise, we have that for all $x, \operatorname{supp}\left(R^{1} R_{x}\right)=\operatorname{supp}\left(R_{x} R^{1}\right)=X$. These conditions imply that $X \subseteq X x \cup\{x\}$ and $X \subseteq x X \cup\{x\}$ for all $x \in X$.

It suffices to show that under these conditions, the subsemigroup of $S$ generated by $X$ is actually a finite group, for then we may conclude by (vi) that $R$ is perfect. This assertion follows at once from the next lemma, which completes the proof of Theorem 1.

LemMa 4. Let $S$ be a semigroup and let $X$ be a finite generating subset of $S$ with at least two elements. Suppose that $X$ satisfies:

$$
X \subseteq X x \cup\{x\} \text { and } X \subseteq x X \cup\{x\} \text {, for all } x \in X .
$$

Then $S$ has an identity element 1 and $S=X \cup\{1\}$ is a finite group. 
Proof. We first show that $S$ has an identity element. Since $X$ has at least two elements, we may choose distinct elements $x$ and $y$ of $X$. Then by (1), there are elements $a, b \in X$ such that $x=a y$ and $y=b x$. From these equations, we conclude that $a b x=x$. But $X \subseteq x X \cup\{x\}$, so that $a b z=z$ for all $z \in X$, and since $X$ generates $S$, we see that $a b$ is a left identity element of $S$. A similar argument shows that $S$ has a right identity element, and therefore a unique two-sided identity element 1.

Let $x \in X$. If $x=a x$ for some $a \in S$, then, as above, $a$ is a left identity for $S$ and therefore $a=1$.

Now let $x \in X$. We will show that $x$ has a left inverse in $X$. Let $z \in X$ be an element different from $x$. By (1), there is a $y \in X$ such that $z=x y$. If $z=y$, then by the above argument we conclude that $x=1$. Otherwise, by (1) again, there is a $w \in X$ such that $y=w z$. Combining these equations, we get $y=w x y$, whence $w x=1$.

The argument of the last paragraph shows that $1 \in X x$. Put $X^{\prime}=X \cup\{1\}$. We have $X^{\prime} x=X x \cup\{x\}$, so from (1) and the fact $1 \in X x$, we see that $X^{\prime} \subseteq X^{\prime} x$. Since $X^{\prime}$ is finite, we must in fact have the equality $X^{\prime} x=X^{\prime}$, and similarly, $x X^{\prime}=X^{\prime}$ for all $x \in X^{\prime}$ (the equations being trivial for $x=1$ ). In particular, this means that the set $X^{\prime}$ is closed under multiplication, and since it contains an identity and left inverses, it forms a group. Finally, $X$ being a generating subset of $S$ ensures that $S=X^{\prime}$ is a finite group.

Note that the converse of Theorem 1 was recently proved by Jespers and Okniński [10]. In fact, they proved a stronger statement that for any perfect semigroup-graded ring (not necessarily having finite support) and for any subgroup $G$ of $S$ (in particular, for $G=\{e\}, e$ an idempotent), the subring $R_{G}=\sum_{g \in G} R_{g}$ is perfect. They proved a similar result for semilocal and semiprimary rings, and the appropriate converse statements are trivially true in the cases of nilpotent rings and PI-rings.

We mention an interesting corollary of Proposition 3, which was proved in [2] using a combinatorial method.

COROLLARY 5. Let $R$ be a semigroup-graded ring whose support is finite and contains no idempotents. Then $R$ is nilpotent.

4. Rings which cannot be regraded. Let $S$ be a semigroup and let $R$ be an $S$-graded ring with finite support. It is conceivable that it is somehow possible to relabel the components of $R$ with elements of another semigroup $T$ in such a way that $R$ becomes a $T$-graded ring, and furthermore so that $T$ is a nicer semigroup: a finite semigroup, or one with no principal factors which are 0 -simple but not completely 0 -simple. If this were always possible, then the methods of [2], [3], [4], [11] would be sufficient to prove all the results of the previous section. In this section, we give some examples of semigroupgraded rings with finite support which cannot be so regraded.

We must first make precise what we mean by regrading a semigroup-graded ring. Let $R$ be a ring graded by a semigroup $S$. Let $T$ be another semigroup. We say that $R$ can be regraded faithfully by $T$ if there is an injective map $\psi: \operatorname{supp}(R) \rightarrow T$ such that $R^{\prime}=R$ is a $T$-graded ring if we put $R_{t}^{\prime}=R_{\psi^{-1}(t)}$ for $t \in \psi(\operatorname{supp}(R))$ and $R_{t}^{\prime}=0$ otherwise. Essentially, we relabel the non-zero homogeneous components of $R$ by some elements of $T$ in such a way that the multiplication of homogeneous elements of $R$ is compatible with the semigroup multiplication in $T$. 
Let us examine this process more closely. Suppose then that we are in the situation outlined above, and $s, t \in S$ are elements such that $R_{s} R_{t} \neq 0$. Then $R_{s} R_{t} \subseteq R_{s t}$. Hence in the ring $R^{\prime}$ we require that $R_{\psi(s)}^{\prime} R_{\psi(t)}^{\prime} \subseteq R_{\psi(s t)}^{\prime}$ and because $R_{\psi(s)}^{\prime} R_{\psi(t)}^{\prime} \neq 0$, we must have $R_{\psi(s t)}^{\prime}=R_{\psi(s) \psi(t)}^{\prime}$ so that $\psi(s) \psi(t)=\psi(s t)$.

We will produce a ring graded by an infinite semigroup, with finite support, which cannot be regraded by a finite semigroup. In fact, the grading semigroup is a group, so this example solves the corresponding question for group graded rings at the same time.

The ring is constructed from an example, due to Dăscălescu, Năstăsescu, del Río, and Van Oystaeyen [7], of a finite subset of an infinite group which cannot be embedded in a finite group in such a way that all products which do not leave the subset are preserved. We first present this example and show that such an embedding into a finite semigroup is also impossible.

EXAMPLE 1. Let $G$ be an infinite simple group which is finitely presented. Such groups exist, see for example [8]. Specifically, let $X$ be a finite set of symbols, let $F$ be the free group on $X$, let $W$ be a finite set of words in $F$, and let $N$ be the normal subgroup of $F$ generated by $W$ such that $F / N \cong G$. For an element $w \in F$ we will write $\bar{w}$ for the image of $w$ in $G$.

Define a subset $A$ of $F$ by

$$
A=\{w \mid w \text { is a subword of an element of } W\} \cup X \cup X^{-1} \cup\{1\},
$$

where we denote the identity element of a group by 1 , and $X^{-1}=\left\{x^{-1} \mid x \in X\right\}$. Note that we include the elements of $W$ itself in $A$. Because $W$ and $X$ are finite, $A$ is finite. Write $\bar{A}$ for the image of $A$ in $G$. We may assume that elements of $X$ are distinct in $G$ and identify $X$ with its image in $G$. Note also that $|X|>1$.

We now show that the subset $\bar{A}$ of $G$ has the property described above.

LeMma 6. There is no injective map $\psi: \bar{A} \rightarrow S$ into a finite semigroup $S$ such that $\psi(\bar{u}) \psi(\bar{v})=\psi(\bar{u} \bar{v})$ whenever $u, v, u v \in A$.

Proof. Suppose there is such a map. We first claim that we can replace $S$ by a group H. For if $g \in \bar{A}$, then $g=g 1=1 g$ so that $\psi(g)=\psi(g) \psi(1)=\psi(1) \psi(g)$, and also $\psi(1) \psi(1)=\psi(1)$. Letting $e=\psi(1)$, we see that $\psi(\bar{A})$ is contained in the monoid $e S e$. Let $w$ be an element of $A$. If $w$ is a subword of an element of $W$, then there are elements $u, v \in A$ such that $u w v$ is an element of $W$ and so $\bar{u} \bar{w} \bar{v}=1$. If $w \in X \cup X^{-1} \cup\{1\}$, then take $u=1$ and $v=w^{-1}$ (which are elements of $A$ ) and we also have $\bar{u} \bar{w} \bar{v}=1$. Furthermore, we have $u w \in A$ in each case. Hence,

$$
e=\psi(1)=\psi(\bar{u} \bar{w} \bar{v})=\psi(\bar{u} \bar{w}) \psi(\bar{v})=\psi(\bar{u}) \psi(\bar{w}) \psi(\bar{v}) .
$$

But $e S e$ is a finite monoid, so left units are also right units and it follows that $\psi(\bar{w})$ is a unit. So $\psi(\bar{A})$ is contained in the group of units $H$ of the monoid $e S e$.

Now, the restriction $\left.\psi\right|_{X}$ induces a group homomorphism $\phi: F \rightarrow H$, since $F$ is a free group generated by $X$. It is straightforward to check that $\phi(w)=\psi(\bar{w})$ for any $w \in A$. In particular, for $w \in W$, we have $\phi(w)=\psi(\bar{w})=\psi(1)=e$. Hence, $\phi(N)=e$ and $\phi$ induces a homomorphism $\bar{\phi}: G \rightarrow H$. But $\bar{A}$ certainly has more than two elements (since $X \subseteq \bar{A}$ ) 
and $\psi$ is injective, so the image of $\psi$ and hence the image of $\bar{\phi}$ are non-trivial. This is not possible, since $G$ is an infinite simple group and $H$ is finite.

This final contradiction shows that there is no such map $\psi$.

We now construct, using the group $G$ of Example 1, a $G$-graded ring with finite support which cannot be faithfully regraded by a finite semigroup.

EXAMPLE 2. Let $n$ be the maximum length of a word in $W$ and let $P$ be a ring with an ideal $Q$ such that $Q^{2 n+1}=0$ but $Q^{2 n} \neq 0$.

Let $R$ be the subring of the group ring $P[G]$ generated by the set

$$
P 1 \cup\left(\bigcup_{x \in X} Q x\right) \cup\left(\bigcup_{x \in X^{-1}} Q x\right)
$$

Since $R$ is generated by homogeneous elements, it is a homogeneous subring of $P[G]$. For $g \in G$, the $g$-component of $R$ is $R_{g}=R \cap P g$.

Let $g \in G$. If $k$ is the length of the shortest product of elements $x_{1}, x_{2}, \ldots, x_{k}$ with each $x_{i} \in X \cup X^{-1}$ such that $g=x_{1} x_{2} \ldots x_{k}$, then $R_{g}=Q^{k} g$. This means that $\operatorname{supp}(R)$ is finite, since $Q^{2 n+1}=0$ and only finitely many elements of $G$ can be written as such products of length less than $2 n+1$.

Let $w \in A$. If $w \in X \cup X^{-1}$, then $R_{\bar{w}}=Q \bar{w}$. If $w=1$, then $R_{\bar{u}}=P 1$. Otherwise, $w$ is a subword of length $k$ of some element of $W$, and by choice of $n, 0 \neq Q^{k} \bar{w} \subseteq R_{\bar{w}}$. Hence $\bar{A} \subseteq \operatorname{supp}(R)$. Furthermore, if $u, v, u v \in A$, then $Q^{k} \bar{u} \subseteq R_{\bar{u}}$ and $Q^{\prime} \bar{v} \subseteq R_{\bar{v}}$ for some $k, l \leq n$, so that $0 \neq Q^{k+l} \bar{u} \bar{v} \subseteq R_{\bar{u}} R_{\bar{v}} \subseteq R_{\bar{u} \bar{v}}$.

If $R$ can be faithfully regraded by a finite semigroup $S$, then there is an injective map $\phi: \operatorname{supp}(R) \rightarrow S$ such that $\phi(g) \phi(h)=\phi(g h)$ whenever $R_{g} R_{h} \neq 0$. But by the above, the restriction of $\phi$ to $\bar{A}$ satisfies the properties of the map $\psi$ of Lemma 6 . Since such a map $\psi$ does not exist, we conclude that $R$ cannot be faithfully regraded by a finite semigroup.

An $S$-graded ring $R$ is strongly graded if $R_{x} R_{y}=R_{x y}$ for all $x, y \in S$. The ring constructed above is not strongly graded; for example, if $x \in X$, then $R_{x} R_{x^{-1}}=Q^{2} 1 \neq R_{1}$. More generally we have the following result.

PROPOSITION 7. Let $R$ be a ring which is strongly graded by a semigroup $S$ and which has finite support. Then $R$ can be faithfully regraded by a finite semigroup.

Proof. Let $I=\left\{x \in S \mid R_{x}=0\right\}$. Because $R$ is strongly graded, $I$ is an ideal of $S$. The canonical map $\psi: S \rightarrow S / I$ induces a faithful regrading of $R$ by the finite semigroup $S / I$.

Our second example is of a ring graded by a 0 -simple semigroup which cannot be regraded by a completely 0 -simple semigroup, or a semigroup with only completely 0 -simple or null principal factors.

EXAMPLE 3. Let $S=\mathscr{C}(p, q)$ be a bicyclic semigroup, that is, the monoid generated by elements $p$ and $q$, subject to the relation $p q=1$ (cf. $[5, \S 12]$ ). Let $I$ be an ideal of a ring $A$ with $I^{3}=0$ but $I^{2} \neq 0$. Let $R$ be the subring of $A[S]$ generated by the homogeneous elements $A 1 \cup I p \cup I q$, and let $R$ inherit the $S$-gradation from $A[S]$.

Suppose that $\psi: \operatorname{supp}(R) \rightarrow T$ is a map that faithfully regrades $R$. Then it is easy to 
see, by considering products of homogeneous components of $R$, that the following identities hold in $T$ :

$$
\begin{gathered}
\psi(1)^{2}=\psi(1), \\
\psi(1) \psi(p)=\psi(p) \psi(1)=\psi(p), \\
\psi(1) \psi(q)=\psi(q) \psi(1)=\psi(q), \\
\psi(p) \psi(q)=\psi(1) \quad \text { and } \quad \psi(q) \psi(p)=\psi(q p) .
\end{gathered}
$$

Write $1=\psi(1), p^{\prime}=\psi(p)$, and $q^{\prime}=\psi(q)$ and let $T^{\prime}=\left\langle p^{\prime}, q^{\prime}\right\rangle$, the subsemigroup of $T$ generated by $p^{\prime}$ and $q^{\prime}$. Because we require $\psi$ to be injective, we have $q^{\prime} p^{\prime} \neq 1$. But this means that $T^{\prime}$ is actually a bicyclic semigroup [5, Lemma 1.31]. Therefore, $T$ cannot be completely 0 -simple (since $T^{\prime}$ contains non-primitive idempotents), and furthermore, the principal factor of $T$ which contains $p^{\prime}$ must contain all of $T^{\prime}$ (since the latter semigroup is simple), and therefore cannot be completely 0 -simple.

ACKNOWLEDGEMENTS. We are grateful to the referee for drawing our attention to [9]. This enabled us to include the class of Jacobson rings in Proposition 3, replacing a weaker result that we had initially proved for Jacobson rings.

\section{REFERENCES}

1. M. Beattie and E. Jespers, On perfect graded rings, Comm. Algebra 19 (1991), 2363-2371. (1993).

2. M. V. Clase, Semigroup graded rings, Ph.D. thesis, Memorial University of Newfoundland

3. M. V. Clase and E. Jespers, Perfectness of rings graded by finite semigroups, Bull. Soc. Math. Bel. Sér. A 45 (1993), 93-102.

4. M. V. Clase and E. Jespers, On the Jacobson radical of semigroup graded rings, J. Algebra 169 (1994), 79-97.

5. A. H. Clifford and G. B. Preston, The algebraic theory of semigroups, two volumes (American Mathematical Society, 1961).

6. M. Cohen and L. H. Rowen, Group graded rings, Comm. Algebra 11 (1983), 1253-1270.

7. S. Dăscălescu, C. Năstăsescu, A. del Río, and F. Van Oystaeyen, Gradings of finite support. Applications to injective objects, J. Pure Appl. Algebra, to appear.

8. G. Higman, Finitely presented infinite simple groups, Notes on Pure Mathematics, vol. 8, I.A. S. Australian National University, (1974).

9. M. Jaegermann and A. D. Sands, On normal radicals, $N$-radicals, and $A$-radicals, $J$. Algebra 50 (1978), 337-349.

10. E. Jespers and J. Okniński, Descending chain conditions and graded rings, J. Algebra, to appear.

11. A. V. Kelarev, On semigroup graded PI-algebras, Semigroup Forum 47 (1993), 294-298.

12. C. Menini, Finitely graded rings, Morita duality and self-injectivity, Comm. Algebra 15 (1987), 1779-1797.

13. C. Menini and C. Năstăsescu, gr-simple modules and gr-Jacobson radical. Applications, Bull. Math. Soc. Sci. Math. R. S. Roumanie (N.S.) 34 (82) (1990), 25-36.

14. C. Menini and C. Năstăsescu, gr-simple modules and gr-Jacobson radical. Applications II, Bull. Math. Soc. Sci. Math. R. S. Roumanie (N.S.) 34 (82) (1990), 125-133.

15. C. Năstăsescu, Some constructions over graded rings: applications, J. Algebra 120 (1989), $119-138$. 
16. D. S. Passman, Infinite crossed products (Academic Press, 1989).

17. L. H. Rowen, General polynomial identities. II, J. Algebra 38 (1976), 380-392.

18. J. F. Watters, Polynomial extensions of Jacobson rings, J. Algebra 36 (1975), 302-308.

M. V. Clase:

Department of Mathematics and Statistics

MCMASTER UNIVERSITY

HAMILTON

ONTARIO

CANADA L8S 4K1

E. JESPERS:

Department of Mathematics and Statistics

Memorial University of Newfoundland

ST. JOHN'S

NEWFOUNDLAND

Canada A1C 5S7

A. DEL Río:

Departamento de Matemáticas

Universidad de Murcia

30071 MURCIA

SPAIN 